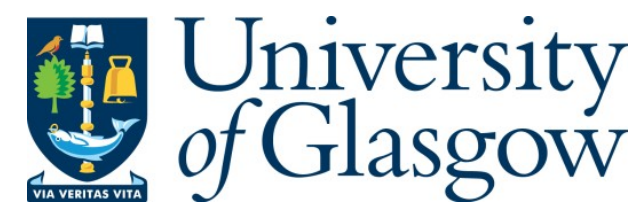

Leuenberger, S. (2014) Total logic. Review of Symbolic Logic, 7 (3). pp. 529-547. ISSN 1755-0203

Copyright $@ 2014$ Cambridge University Press

A copy can be downloaded for personal non-commercial research or study, without prior permission or charge

Content must not be changed in any way or reproduced in any format or medium without the formal permission of the copyright holder(s)

When referring to this work, full bibliographic details must be given

http://eprints.gla.ac.uk/93863/

Deposited on: 26 A ugust 2014

Enlighten - Research publications by members of the U niversity of Glasgow http://eprints.gla.ac.uk 


\section{Total Logic*}

\section{Stephan Leuenberger}

February 14, 2014

Abstract: A typical first stab at explicating the thesis of physicalism is this: physicalism is true iff every fact about the world is entailed by the conjunction of physical facts. The same holds, mutatis mutandis, for other hypotheses about the fundamental nature of our world. But it has been recognized that this would leave such hypothese without the fighting chance that they deserve: certain negative truths, like the truth (if it is one) that there are no angels, are not entailed by the physical facts, but nonetheless do not threaten physicalism. A plausible remedy that has been suggested by Jackson and Chalmers is that physicalism boils down to the thesis that every truth is entailed by the conjunction of the physical facts prefixed by a "that's it" or "totality" operator. To evaluate this suggestion, we need to know what that operator means, and - since the truth of physicalism hinges on what is entailed by a totality claim - what its logic is. That is, we need to understand the logic of totality, or total logic. In this paper, I add a totality operator to the language of propositional logic, and present a model theory for it, building on a suggestion by Chalmers and Jackson. I then prove determination results for a number of different systems.

Author email: stephan.leuenberger@glasgow.ac.uk

Author homepage: http://www.gla.ac.uk/schools/humanities/staff/stephanleuenberger

* Many thanks to audiences in London, Neuchâtel, Tübingen, and Auckland, where some of this material was presented. Special thanks to Jan Plate for his commentary in Tübingen, to Peter Fritz for discussion, to David Chalmers for correspondence, and to two anonymous referees for extremely helpful comments. 


\section{Entry by entailment and negative truths}

In inquiry, we want to find the truth about the world. But we may also want to know, for a given truth, whether it is comprehensive: whether it gives a full account of the world, as it were. To take an example that has figured prominently in philosophical debates: given a complete physical description of the world, it is natural to wonder whether it leaves out something, or whether every truth about the world is, in some sense, already contained in it. Physicalism is committed to rule out the first alternative. The same holds, mutatis mutandis, for alternative hypotheses about the world, such as the idealist view that some mental description of a world is comprehensive, or the dualist view that while some physical-cummental description is comprehensive, no purely physical or purely mental one is.

What, in more precise terms, is it for a truth to be comprehensive? According to one influential approach, it is a matter of entailing all truths. Frank C. Jackson observed that a defender of a serious metaphysical view faces a "placement problem" or "location problem": to show that every feature of our world "[has] a place in the account some serious metaphysics tells of what our world is like" (Jackson (1998, p. 5); see also Jackson (1994)). On his view - the so-called "entry by entailment" doctrine - a feature has such a place "if the feature is entailed by the account told in the terms favoured by the metaphysics in question" (Jackson (1998, p.5)). Similarly, Jackson's joint paper with David J. Chalmers (Chalmers and Jackson (2001)) as well as Chalmers (2012) are concerned with identifying truths $A$ such that for every truth $B$, the material conditional $A \rightarrow B$ is a priori-a relation closely akin to entailment.

This idea faces a simple problem: typical candidates for comprehensive truths do not entail certain negative truths. So, for example, the conjunction of all physical truths does not entail the truth-assuming it is one - that there are no angels. Presumably, there is an angel-ridden possible world that is physically exactly like ours. In response, Jackson and Chalmers modify their account of what it takes for a truth $A$ to be comprehensive. It is not necessary that $A$ by itself entails every truth. Rather, $A$ together with a statement to the effect that $A$ is a total truth - in a sense to be specified - needs to entail every truth. To return to the troublesome example: the complete physical truth about the world, together with the truth that that truth is total, entails that there are no angels. In an angel-ridden world, no physical truth is total, after all. (Jackson (1994), Jackson (1998)) 
The added conjunct has various names in the literature: "totality statement", "that's all clause", or "stop clause". We can take such a statement or clause to be the result of applying an operator to another sentence. The operator - to be called a "totality operator" - may be pronounced "and that's it". Applied to a sentence $A$, it yields another sentence, " $A$, and that's it". I shall use the symbol $T$ for it. To make scope distinctions more perspicuous, I shall prefix rather than suffix it to the sentence it operates on. ${ }^{1}$

An account of the semantics of such a totality operator is implicit in the joint paper Chalmers and Jackson (2001). Chalmers then invoked a totality operator in his most recent presentation of the zombie argument against physicalism (Chalmers (2010)), and in his Chalmers (2012). ${ }^{2}$ Due to the influence of these works, the totality operator has become reasonably familiar to many philosophers. Still, its logic and semantics - the consequences of the account implicit in Chalmers and Jackson (2001) - have hardly received any discussion. This paper aims to remedy this, and provide us with a fuller understanding of the workings of this operator.

The formal tools used are taken from modal logic. Since the study of the logic of modality is called "modal logic", I shall call the study of the logic of totality "total logic".

I start by taking a closer look at the Chalmers-Jackson account of the semantics of $T$ (section 2). Next, I introduce formal "totality models" (section 3). The class of all totality models is shown to determine a simple base system of total logic, called ' $C$ ' (section 4). Finally, I discuss extensions of the base system (sections 5 and 6).

\section{The metaphysics of outstripping}

As mentioned above, a sketch for a semantics of the totality operator is offered in Chalmers and Jackson (2001). They specify the truth-conditions of the operator in terms of the notion of a world's being minimal in a certain class of worlds, and

\footnotetext{
${ }^{1}$ My regimentation of "that's-it" talk with an operator does not commit me to the claim that there is such an operator in English, nor to the claim that if there is such an operator, it is conceptually primitive, rather than understood in terms of some "totality predicate". Thanks to Kevin Mulligan for discussion on this point.

${ }^{2}$ Totality facts - as opposed to totality statements and totality operators - had been discussed already in Armstrong (1989).
} 
define minimality, in turn, in terms of a relation of outstripping. They use ' $P$ ' to denote a complete physical description of the world, and formulate their solution to the problem of negative truths as follows:

This loophole can be closed by conjoining to $P$ a "that's-all" statement $T$, asserting that our world is a minimal world satisfying $P$. Intuitively, this statement says that our world contains what is implied by $P$, and only what is implied by $P$. . [A] minimal $P$-world is a $P$-world that outstrips no other $P$-world. It is plausible that no world containing angels is a minimal $P$-world: for any $P$-world containing angels, there is an angel-free $P$-world that it outstrips. So $P \& T$ implies that there are no angels. (Chalmers and Jackson (2001, p. 317))

While an account of a totality operator is implicit, Chalmers and Jackson are explicitly concerned with a separate statement $T$, read as "that's all". The word "that" anaphorically refers to $P$, so that the logical form of $T$ is more perspicuously represented by writing $T(P)$. In their semantics, $T(P)$ entails $P$. For that reason, the first conjunct in $P \& T(P)$ is redundant. I shall thus write $T(P)$ or simply $T P$ instead of $P \& T$.

The Chalmers-Jackson theory explains the totality operator in terms of possible worlds, and a certain relation of outstripping. If we wish to study the logic of totality, we do not need to know the nature of this relation of outstripping, as opposed to its formal properties. The situation is analogous to the one in modal logic: when doing logic, we can be unconcerned about what kind of entities possible worlds are, and what it is for one world to be accessible from another. We can treat possible worlds just as any members of some set, and only need assumptions about the formal features of the accessibility relation. Still, as philosophers, we may wish to know something about the metaphysics of possible worlds, accessibility, and in our case outstripping. Moreover, we will not be able to ignore metaphysical questions if we wish to determine what the right logic of totality is.

Chalmers and Jackson give an account of outstripping in terms of parthood and duplication:

More formally, we can say that world $w_{1}$ outstrips world $w_{2}$ if $w_{1}$ contains a qualitative duplicate of $w_{2}$ as a proper part and the reverse is not the case. (Chalmers and Jackson (2001, p. 317)) 
For future reference, I shall call the biconditional that follows from this definition "CJ-Outstripping". 3

CJ-Outstripping $w$ outstrips $w^{\prime}$ iff some proper part of $w$ duplicates $w^{\prime}$, but no proper part of $w^{\prime}$ duplicates $w$.

For our study of the logic of totality, it will be important what formal features the relation of outstripping has. It is immediate from the formulation of the account that outstripping is asymmetric. Furthermore, we can appeal to the following principle to show that outstripping is transitive.

DupDec If $x$ and $x^{\prime}$ are duplicates and $y$ is a proper part of $x$, then there is a proper part $y^{\prime}$ of $x^{\prime}$ that is a duplicate of $y$.

DupDec captures the plausible thought that two things with non-matching decompositions into parts are not duplicates. For example, none of my duplicates will be an extended mereological simple if I myself have proper parts.

To show that DupDec entails the transitivity of outstripping, suppose that $w$ outstrips $w^{\prime}$, and $w^{\prime}$ outstrips $w^{\prime \prime}$. Then there is a proper part $v$ of $w$ that duplicates $w^{\prime}$, and a proper part $v^{\prime}$ of $w^{\prime}$ that duplicates $w^{\prime \prime}$. Then DupDec guarantees that $v$ has a proper part $t$ that duplicates $v^{\prime}$. Since proper parthood is transitive, $t$ is a proper part of $w$. Since duplication is transitive, $t$ duplicates $w^{\prime \prime}$. So some proper part of $w$ duplicates $w^{\prime \prime}$. Suppose now, for reductio, that a proper part $v^{\prime \prime}$ of $w^{\prime \prime}$ duplicates $w$. Then by DupDec, it follows that $v^{\prime \prime}$ has a proper part $t^{\prime \prime}$ that duplicates $v$. Since duplication is transitive, $t^{\prime \prime}$ duplicates $w^{\prime}$. Since proper parthood is transitive, $t^{\prime \prime}$ is a proper part of $w^{\prime \prime}$. Hence some proper part of $w^{\prime \prime}$ is a duplicate of $w^{\prime}$, and $w^{\prime}$ does not outstrip $w^{\prime \prime}$, contrary to our assumption.

So outstripping is asymmetric and transitive, and is thus a partial order. One might think that the clause "but no proper part of $w^{\prime}$ duplicates $w$ " is redundant, and that the statement of the mereological account could thus be simplified. Indeed, in his most recent presentation of this account, Chalmers drops that clause, and appears to suggest that he is still defining a partial order: "[W]e have a partial ordering among worlds. We might say that world $w_{1}$ outstrips another world $w_{2}$ if

\footnotetext{
${ }^{3}$ I have changed "qualitative duplicate" to "duplicate" (as has, incidentally, Chalmers in his formulation in Chalmers (2012, p. 151)). On some accounts, a qualitative duplicate is a duplicate simpliciter. But for the purposes of the logic of totality, I wish to remain neutral on this metaphysical issue.
} 
$w_{1}$ includes a duplicate of $w_{2}$ as a proper part" (Chalmers (2012, p. 151)). Call the relation thus defined outstripping*. It turns out that given DupDec, outstripping* is co-extensional with outstripping if and only if the following principle holds:

DupPart No world duplicates any of its proper parts. ${ }^{4}$

Moreover, outstripping* is not a partial order, if DupPart is false. ${ }^{5}$

However, DupPart is arguably false. Consider a world of one-way infinite recurrence. The world minus the first epoch is a proper part of the whole world. Arguably, though, that proper part is a duplicate of the whole world.

So much for the formal features that outstripping has, on the Chalmers-Jackson account. It remains to clarify what the pertinent notion of parthood is. On one popular conception, parts are spatiotemporal parts - a part occupies a subregion of the region occupied by the whole. But on this conception, CJ-Outstripping does not give the desired result in the case of the angels. Suppose that angels are non-physical, but still in spacetime. Then a physical duplicate of the actual world in which there are angels may have no proper part that is a duplicate simpliciter of the actual world. For proper parts of the world would involve proper parts of its spacetime, and those may fail to be duplicates of the actual world. So the account needs objects to have non-spatiotemporal parts. Arguably, a one-proton world outstrips a one-neutron world. Simplifying the physics a bit: the proton has charge and the neutron has not, while they agree on all other fundamental properties. Hence a proton needs to have a proper part that duplicates a neutron, for example - namely the part we get after subtracting electric charge. Depending on the conception of properties adopted, that part may be itself a property, or it may be a bearer of a property.

This concludes my sketch of the metaphysical background to the logical issues to be discussed in the rest of the paper. I will have occasion to return to the

\footnotetext{
${ }^{4}$ To verify that the co-extensionality of these relations entails DupPart, suppose that the latter is false. Then some $w$ duplicates its own proper part $v$. It follows that $w$ outstrips* itself, but does not outstrip itself. For the converse entailment, suppose now that $w$ outstrips* but does not outstrip $w^{\prime}$. Then some proper part $v$ of $w$ duplicates $w^{\prime}$. But also, some proper part $v^{\prime}$ of $w^{\prime}$ duplicates $w$. By DupDec, $v$ has a proper part $t$ that duplicates $v^{\prime}$. But because both proper parthood and duplication are transitive, $t$ is a proper part of $w$ and duplicates $w$. So DupPart fails.

${ }^{5}$ If DupPart is false, some world outstrips* itself, and so outstripping* is not a strict partial order. But it is not a weak partial order either, since it is not reflexive.
} 
Chalmers-Jackson account of outstripping briefly in the next section, and again in section 6 .

\section{Totality models}

According to the modified entry by entailment account, we need to ask whether $T(A)$ entails every truth in order to evaluate whether some truth $A$ is comprehensive. Logic - on at least one important conception - studies the relation of entailment. This gives us a reason to be interested in the logic of $T$.

A full account of the logic of $T$ would include its interaction with various other operators - modal and epistemic ones, for example - and with quantifiers, including, presumably, propositional quantifiers. In this paper, I shall only treat a very small fragment of the logic of $T$ : its interaction with the connectives of propositional logic, and its behaviour under iteration. I hope that this will provide a sound basis for further and more complex investigations.

Accordingly, I shall here work with a simple formal language, which I call $L_{T}$. Basically, $L_{T}$ is the language of propositional modal logic, except that it has a totality operator $T$ instead of the necessity operator $\square$. The sentences of $L_{T}$ can be specified by the usual formation rules: (i) all atomic sentences $p_{0}, p_{1}, \ldots$ and $\perp$ are $L_{T}$-sentences; (ii) if $A$ and $B$ are $L_{T}$-sentences, so are $\neg A, A \wedge B, A \vee B$, $A \rightarrow B, A \leftrightarrow B$, and $T A$; and (iii) nothing not formed in accordance with (i) and (ii) is an $L_{T}$-sentence.

One limitation of the language $L_{T}$ deserves discussion: it does not allow the formation of sentences that are infinitely long. In the present context, this may appear to be a serious shortcoming. For candidate sentences to which $T$ may potentially be truly applied are typically infinitary. For all we know, a complete physical description of the world must specify the value of certain fields at continuum-many spacetime-points. This may prompt the worry that the relevant logical structure of philosophical arguments involving totality-operators cannot be captured in $L_{T} \cdot{ }^{6}$

However, it is unclear whether the validity of those arguments in any way turns on logical issues related to infinity. Nothing stops us from taking a complete physical description of the world as the interpretation of an atomic sentence of $L_{T}$. Infinity can thus be "hidden away" in a syntactically simple item. Still, it would be

\footnotetext{
${ }^{6}$ Thanks to Graham Priest for raising this worry.
} 
an interesting project to explore a language in which $T$ applies to a set of sentences (of arbitrary cardinality) and forms a sentence. That project is beyond the scope of the present paper, however.

I shall now specify a model theory for this language. Perhaps unsurprisingly, the models to be used in interpreting $L_{T}$ involve possible worlds and a relation of outstripping. As in modal logic, it will be convenient to distinguish between frames, which abstract from the interpretation of atomic sentences, and models, which do take that interpretation into account.

A totality frame $\mathcal{F}$ is a pair $\langle W,<\rangle$, where $W$ is a set and $<$ a binary relation on $W$. A totality model $\mathcal{M}$ is a triple $\langle W,<, I\rangle$, where $\langle W,<\rangle$ is a totality frame and $I$ an assignment, taken to be a function that assigns a subset of $W$ to every non-negative integer.

Next, we define what it is for a sentence $A$ of $L_{T}$ to be true at a world $w$ in a model $\mathcal{M}$ - in symbols, $\models_{w}^{\mathcal{M}} A$. Atomic sentences are indexed by the non-negative integers, and $p_{j}$ is true in a world in a model iff that world belongs to the image of $j$ under $I: I: \models_{w}^{\mathcal{M}} p_{j}$ iff $w \in I(j)$. For sentences with the connectives $\neg, \wedge, \vee, \rightarrow, \leftrightarrow$, the clauses are those familiar from propositional logic. The interesting evaluation clause is the one for $T$. Following Chalmers and Jackson, we specify it as follows:

Definition 1. $\vDash_{w}^{\mathcal{M}} T A$ iff $\models_{w}^{\mathcal{M}} A$ and for all $w^{\prime}$ such that $w^{\prime}<w, \not_{w^{\prime}}^{\mathcal{M}} A$.

So $T A$ is true at $w$ iff $w$ is a minimal one among the worlds where $A$ is true.

Using the Chalmers-Jackson account of outstripping, the condition that if $T A$ is true in $w, A$ is not true in any $w^{\prime}$ outstripped by $w$ can be heuristically motivated as follows: if $A$ is true in such a $w^{\prime}$, then $A$ does not describe more than a proper part of $w$ - the one that is a duplicate of $w^{\prime} .^{7}$ Let $v$ be such a part. As a consequence of a relatively uncontroversial mereological principle - called "Weak Supplementation" or sometimes just "Supplementation" in the literature - there is a part $u$ of $w$ that does not overlap $v{ }^{8}$ But then, it seems, $A$ fails to describe $u$. Hence " $A$, and that's it" is intuitively false.

Suppose that $A$ correctly describes a proper part of the world, but not the whole world $-A$ is true and $T A$ false. Then, given a certain interpreted language,

\footnotetext{
${ }^{7}$ Since more than one $A$-world may be outstripped by $w$, and since $w$ may have duplicate proper parts, there is not, in general, a unique proper part of $w$ that duplicates an $A$-world outstripped by $w$.

${ }^{8}$ For mereological notions, see Varzi (2012), for example.
} 
there is no guarantee that the rest of the world can be described as well. Indeed, no truth logically stronger than $A$ may be expressible. So there is no guarantee that $T B$ is true for any sentence $B$ in the language. For example, all the atomic sentences might be interpreted to mean that there is strictly more than $1 g$ of mass in the universe. Then no world with massive objects is going to be exhaustively described by any truth in that language. Switching back to talk of models instead of interpreted languages, I shall call a world $w$ inexhaustible in a model $\mathcal{M}$ iff $\forall_{w}^{\mathcal{M}} T A$ for all $A \in L_{T}$. This concept will be useful in later sections.

We are now in a position to define validity of sentences of $L_{T}$ relative to our model theory. The definitions are standard ones: $A$ is valid in a model $\mathcal{M}=\langle W,<$ $, I\rangle$ iff it is true in every $w \in W$; it is valid in a frame iff it is valid in all models on that frame; it is valid in a class of frames (models) iff it is valid in every member of that class.

Occasionally, I shall call the model theory just described a "semantics", even though this might be misleading. For it does not, by any standard, specify the meaning of the operator $T$. The intended meaning is "and that's it", but the model theory allows for many other interpretations, besides the totality interpretation.

For example, we might give $T$ a primacy interpretation: ' $T A$ ' means that $A$ is true for the first time. On that interpretation, the symbol $<$ should be thought of as standing for the earlier-than relation. Conversely, we might give $T$ an ultimacy interpretation: ' $T A$ ' means that $A$ is true for the last time. We may note that on the primacy and the ultimacy interpretation, the operator $T$ is definable in the language of tense logic. 'For the first time, $A$ ' is true at an instant just in case both $A$ and $H \neg A$, meaning "it has always been the case that $\neg A$ ", are true. "For the last time, $A^{\prime}$ is true at an instant just in case both $A$ and $G \neg A$, meaning "it will always be the case that $\neg A$ ", are true.

Suppose that we used the relation of outstripping to define an operator $O$ as follows:

Definition 2. $\vDash_{w}^{\mathcal{M}} O A$ iff for all $w^{\prime}$ such that $w^{\prime}<w, \vDash_{w^{\prime}}^{\mathcal{M}} A$.

Then we could, in analogy to the tense-logical case, define $T A$ by $A \wedge O \neg A$.

Given the Chalmers-Jackson account of outstripping, $O A$ means that $A$ is true in all worlds that duplicate a proper part of ours, but do not have a proper part that is duplicated by our world. We may note that the evaluation clause of $O$ is 
the same as the one for $\square$ in standard modal logic, except that the role of the accessibility relation $R$ is played by the converse of $<$.

Together with the decidability of the set of sentences valid in all Kripke modelsa basic result in modal logic (see Lemmon and Scott (1977, p. 44) or any other textbook) - the definability of $T$ in terms of $O$ entails that the set of sentences valid in all totality models is also decidable. For we can easily translate any $A \in L_{T}$ into an $A^{\prime} \in L_{\square}$ such that $A$ is valid in all totality models iff $A^{\prime}$ is valid in all Kripke frames, and then apply a decision procedure to $A^{\prime}$.

Moreover, it follows from these observations about temporal interpretations of $L_{T}$ that the basic logic of "and that's it" is the same as the basic logic of "for the first time" and of "for the last time". By "basic logic", I here mean the logic we get without imposing constraints on the admissible frames, and without considering the relationship of $T$ to other operators.

\section{The base system of total logic}

After giving the model theory, I shall now specify a formal system. The aim is to axiomatize the class of $L_{T}$-sentences that are valid in all totality models, or equivalently, valid in all totality frames.

We have seen that the operator $T$ can be defined in terms of $O$. If it were also possible to define $O$ in terms of $T$, then the task of axiomatization would be easy. I have remarked that the evaluation clause for $O$ in totality models is the same as the evaluation clause for $\square$ in the standard Kripke semantics (modulo relabelling). Sound and complete axiomatizations of $\square$ relative to Kripke models are well-known. If $O A$ were definable in $L_{T}$, we would obtain a sound and complete axiomatiztion of the class of $L_{T^{-}}$-sentences valid in all totality models simply by substituting an $L_{T^{-}}$ definiens for $O A$ in the system $\boldsymbol{K}$, which is a sound and complete axiomatization of the logic of $O$.

However, $O$ cannot be defined in terms of $T$, as I shall now prove. It is a straightforward consequence of Definition 1 that if $w<w$, then $\neg T A$ is true in $w$ for every $A$. Likewise $\neg T A$ is true for all $A$ in worlds that have an infinite chain of worlds satisfying a certain condition below them. To make this precise, say that $W^{\prime} \subseteq W$ is a chain in $\mathcal{M}$ iff $W^{\prime}$ is totally ordered by $<$. Say that a chain $W^{\prime}$ is unending if for every $w \in W^{\prime}$ there is $w^{\prime} \in W^{\prime}$ such that $w^{\prime}<w$; and that $W^{\prime}$ is 
homogeneous if (i) for all $w, w^{\prime} \in W^{\prime}, j \in \mathbb{N}, w \in I(j)$ iff $w^{\prime} \in I(j)$, and (ii) for all $w, w^{\prime} \in W^{\prime}$ and $w^{\prime \prime} \in W \backslash W^{\prime}, w<w^{\prime \prime}$ iff $w^{\prime}<w^{\prime \prime}$, and $w^{\prime \prime}<w$ iff $w^{\prime \prime}<w^{\prime}$.

Lemma 1. If $W^{\prime}$ is a homogeneous unending chain in $\mathcal{M}$, then for all $w, w^{\prime} \in W^{\prime}$ and all $A, \models_{w}^{\mathcal{M}} A$ iff $\models_{w^{\prime}}^{\mathcal{M}} A$.

Proof. By induction on the length of formulas. The key step concerns $A=T B$. If $\models_{w}^{\mathcal{M}} T B$, then $\models_{w}^{\mathcal{M}} B$, and $\models_{w^{\prime}}^{\mathcal{M}} B$ by the induction hypothesis. Moreover, $\nvdash_{v}^{\mathcal{M}} B$ for all $v<w$. Since $w$ is part of an unending chain, there is such a $v \in W^{\prime}$. Pick $v^{\prime} \in W^{\prime}$ with $v^{\prime}<w^{\prime}$. By the induction hypothesis applied to $v$ and $v^{\prime}, \not_{v^{\prime}}^{\mathcal{M}} B$. So $\models_{w^{\prime}}^{\mathcal{M}} T B$. If $\nvdash_{w}^{\mathcal{M}} T B$, similar reasoning establishes that $\nvdash_{w^{\prime}}^{\mathcal{M}} T B$.

Lemma 2. If $W^{\prime}$ is a homogeneous unending chain in $\mathcal{M}$, and $w \in W^{\prime}$, then $\vDash_{w}^{\mathcal{M}} \neg T A$.

Proof. If $\forall_{w}^{\mathcal{M}} A$, then $\vDash_{w}^{\mathcal{M}} \neg T A$ by Definition 1. So suppose $\vDash_{w}^{\mathcal{M}} A$. Since $w \in W^{\prime}$ and $W^{\prime}$ is unending, there is $w^{\prime} \in W^{\prime}$ such that $w^{\prime}<w$. Since $W^{\prime}$ is homogeneous, $\vDash_{w^{\prime}}^{\mathcal{M}} A$ by Lemma 1 . Hence $\vDash_{w^{\prime}}^{\mathcal{M}} \neg T A$.

To prove the indefinability of $O$ in $L_{T}$, I shall construct two models with identical homogeneous unending chain. In one of the models, there is a further world outstripped by every world in the chain. Then there are sentences containing $O$, but no $L_{T}$-sentences, whose truth-value at worlds in the chain is sensitive to what is true at that further world. More formally:

Theorem 1. $O$ is not definable in $L_{T}$ relative to the class of totality models.

Proof. Define $\mathcal{M}$ and $\mathcal{M}^{\prime}$ as follows, where $W=\left\{w_{i}: i \in \mathbb{N}\right\},<=\left\{\left\langle w_{i}, w_{j}\right\rangle\right.$ : $\left.i>_{\mathbb{N}} j\right\}, I$ a function that maps every natural number to the empty set, and $I^{\prime}$ a function defined by $I^{\prime}(0)=\{\omega\}$ and $I^{\prime}(i)=\emptyset$ for all $i \neq 0$ :

- $\mathcal{M}=\langle W,<, I\rangle$

- $\mathcal{M}^{\prime}=\left\langle W \cup\{\omega\},<\cup\{\langle\omega, i\rangle: i \in \mathbb{N}\}, I^{\prime}\right\rangle$

Clearly $\vDash_{w_{0}}^{\mathcal{M}} O \neg p_{0}$ and $\nvdash_{w_{0}}^{\mathcal{M}^{\prime}} O \neg p_{0}$. However, for every $A \in L_{T}, \vDash_{w_{0}}^{\mathcal{M}} A$ iff $\vDash_{w_{0}}^{\mathcal{M}^{\prime}}$ $A$. This is proved by induction on the length of formulas. For atomic $p_{i}$, the claim follows immediately from the definition of $I$ and $I^{\prime}$. The induction steps for the usual propositional connectives are straightforward. For $T A$, the claim 
follows immediately from Lemma 2 and the fact that $w_{0}$ belongs to a homogeneous unending chain in both $\mathcal{M}$ and $\mathcal{M}^{\prime}$.

Since $T$ can be defined in terms of $O$, the language $L_{T}$ is expressively weaker than $L_{O}$, which is like $L_{T}$ except that it contains $O$ in place of $T$. Hence we cannot piggy-back directly on the work done in modal logic.

To define axiomatic systems of total logic, I shall start with three axiom schemas:

(A0) All tautologies.

(A1) $T A \rightarrow A$

(A2) $T A \wedge T B \rightarrow T(A \vee B)$

All $L_{T}$-instances of (A0), (A1), and (A2) are valid in all totality models. In the case of $(\mathrm{A} 0)$, this is a consequence of the fact that the evaluation clauses for the usual propositional connectives - which I have not explicitly specified - are the standard ones of classical propositional logic. For (A1), suppose that $\vDash_{w}^{\mathcal{M}} T A$. Then by Definition $1, \vDash_{w}^{\mathcal{M}} A$. Hence by the standard clause for $\rightarrow, \models_{w}^{\mathcal{M}} T A \rightarrow A$. For (A2), suppose that $\vDash_{w}^{\mathcal{M}} T A \wedge T B$. Then $\vDash_{w}^{\mathcal{M}} T A$, and hence $\vDash_{w}^{\mathcal{M}} A$, and therefore $\vDash_{w}^{\mathcal{M}} A \vee B$. Let $w^{\prime}$ be such that $w^{\prime}<w$. Then neither $\vDash_{w^{\prime}}^{\mathcal{M}} A$ nor $\vDash_{w^{\prime}}^{\mathcal{M}} B$, and hence $\not_{w^{\prime}}^{\mathcal{M}} A \vee B$. By Definition 1, it follows that $\models_{w}^{\mathcal{M}} T(A \vee B)$

Next, we consider two rules of inference (' $\vdash A$ ' expresses that $A$ is a thesis):

RMP $\quad$ If $\vdash A \rightarrow B$ and $\vdash A$, then $\vdash B$.

RIM If $\vdash A \rightarrow B$, then $\vdash A \rightarrow(T B \rightarrow T A)$.

'RMP' stands for "Rule of Modus Ponens", and does not require comment. 'RIM' stands for "Rule of Inverse Monotonicity". In modal logic, the Rule of Monotonicity says that if $A \rightarrow B$ is a thesis, then so is $\square A \rightarrow \square B$. The name "Inverse Monotonicity" might suggest the rule that if $A \rightarrow B$ is a thesis, then so is $T B \rightarrow T A$. That rule has some prima facie plausibility: if $B$ is complete enough a description for $T A$ to be true, and $A$ is logically stronger than $B$, then $A$ is also complete enough a description of the world for $T A$ to be true. On reflection, however, it is clear that $T B \rightarrow T A$ is only guaranteed if $A$ is also true. This proviso has been taken into account in the formulation of RIM above. 
RIM preserves validity in all totality models. For $\mathcal{M}=\langle W,<, I\rangle$, suppose that $\vDash^{\mathcal{M}} A \rightarrow B$. Let $w \in W$ be such that $\vDash_{w}^{\mathcal{M}} A$ and $\vDash_{w}^{\mathcal{M}} T B$. Then for $w^{\prime}$ such that $w^{\prime}<w, \nvdash_{w^{\prime}}^{\mathcal{M}} B$. Together with $\models_{w^{\prime}}^{\mathcal{M}} A \rightarrow B$, this entails that $\nvdash_{w^{\prime}}^{\mathcal{M}} A$. Since $w^{\prime}$ was chosen arbitrarily, $\vDash_{w}^{\mathcal{M}} T A$.

Let a system of total logic be any class of $L_{T}$-sentences that includes all instances of (A0)-(A2), and that is closed under RMP and RIM. Let $\boldsymbol{C}$ be the smallest system of total logic. ${ }^{9}$ We have already shown that every thesis of $\boldsymbol{C}$ is valid in every totality frame. So $\boldsymbol{C}$ is sound for the class of all totality frames.

We can now derive further rules that are admissible in any system of total logic. I shall omit the proofs, which are straightforward but occasionally tedious. The rules can be called, respectively, "Rule of Propositional Logic", "Rule of Equivalence", and "Rule of Replacement":

RPL If $\vdash A_{1}, \ldots, \vdash A_{n}$ and $B$ is a $P L$-consequence of $A_{1}, \ldots, A_{n}$, then $\vdash B$.

$\mathrm{RE} \quad$ If $\vdash A \leftrightarrow B$ then $\vdash T(A) \leftrightarrow T(B)$.

RRep $\quad$ If $\vdash B \leftrightarrow B^{\prime}$, then $\vdash A\left[B^{\prime}, B\right] \leftrightarrow A$.

In RRep, ' $A\left[B^{\prime}, B\right]$ ' is any formula that can be obtained from $A$ by replacing $B$ in one or more places with $B^{\prime}$.

Next, I shall list a few schemas all of whose instances are theses of $\boldsymbol{C}$, and thus of every system of total logic.

(T1) $T A \rightarrow T T A$

(T2) $T A \leftrightarrow T T A$

To prove that (T1) is a thesis in every system of total logic, we note that by (A1), $\mathrm{RIM}$, and RMP, $T A \rightarrow(T A \rightarrow T T A)$ is a thesis, from which the result follows by RPL. The thesishood of (T2) follows immediately from that of (T1) and (A1).

A number of other theses can be derived very quickly using RIM. They are all variations on the idea that if something is a total truth, then any truth that is logically stronger is so too:

(T3) $T A \wedge B \rightarrow T(A \wedge B)$

\footnotetext{
${ }^{9}$ It is easy to verify that this is a legitimate definition. The intersection of all systems of total logic is itself a system of total logic, and is a subset of every system of total logic.
} 
To derive this, note that by $\mathrm{RPL}(\mathrm{n}=0), \vdash A \wedge B \rightarrow A$. By RIM, $\vdash A \wedge B \rightarrow$ $(T A \rightarrow T(A \wedge B))$. Also $\vdash T A \rightarrow A$ (Axiom A1). The result follows with RPL.

(T4) $T(A \vee B) \wedge A \rightarrow T A$

For by an instance of (T3), $\vdash T(A \vee B) \wedge A \rightarrow T((A \vee B) \wedge A)$. By RPL,$\vdash$ $((A \vee B) \wedge A) \leftrightarrow A$. With RE, (T4) can be derived.

(T5) $T(A \vee B) \wedge \neg A \rightarrow T B$

(T6) $T(A \vee B) \rightarrow T A \vee T B$

For (T5), note that by (T3), $\vdash T(A \vee B) \wedge \neg A \rightarrow T((A \vee B) \wedge \neg A)$. Since $\vdash(A \vee B) \wedge \neg A \leftrightarrow B$, the result follows with REP. Thesis (T6) follows, using RPL, from (T4) and (T5).

Further, we can generalize (A2) to any finite number of conjuncts, and we can derive that $T$ distributes over conjunction.

(T7) $T A_{1} \wedge \ldots \wedge T A_{n} \rightarrow T\left(A_{1} \vee \ldots \vee A_{n}\right)$

(T8) $T A_{1} \wedge \ldots \wedge T A_{n} \rightarrow T\left(A_{1} \wedge \ldots \wedge A_{n}\right)$

For (T7), we use $n-1$ instances of (A2) and RPL (plus a tacit convention of dropping brackets). Repeated application of (A1) and (T3) allows us to derive (T8).

I shall now show that the system $\boldsymbol{C}$ is complete as well as sound for the class of totality models.

Definition 3. Given a system $S$ of total logic, let $\mathcal{M}_{S}$ be $\langle W,<, I\rangle$, where

1. $W_{S}=\left\{w: w\right.$ a maximal $S$-consistent set of sentences of $\left.L_{T}\right\}$.

2. $<_{S}=\left\{\left\langle w^{\prime}, w\right\rangle\right.$ : for all $A \in L_{T}, A \notin w^{\prime}$ whenever $\left.T A \in w\right\}$.

3. $I_{S}(j)=\left\{w: p_{j} \in w\right\}$.

It is obvious that for any system $S, \mathcal{M}_{S}$ is a totality model.

In light of the following result, $\mathcal{M}_{S}$ deserves to be called a "canonical model" for system $S$.

Lemma 3. For every $w \in W_{S}$ and every $A, A \in w$ iff $\models_{w}^{\mathcal{M}_{S}} A$. 
Proof. By induction on the complexity of $L_{T}$-sentences. For an atomic sentence $p_{j}, p_{j} \in w$ iff $w \in I_{S}(j)$ iff $\vDash_{w}^{\mathcal{M}_{S}} p_{j}$. The induction steps for the connectives of propositional logic are straightforward.

Suppose $T A \in w$. By (A1), TA $\rightarrow A \in w$, and by RPL, $A \in w$. By the induction hypothesis, $\models_{w}^{\mathcal{M}_{S}} A$. Let $w^{\prime}<_{S} w$. Then by Definition 3.2, $A \notin w^{\prime}$. By the induction hypothesis, $\not_{w^{\prime}}^{\mathcal{M}_{S}} A$. Hence $\vDash_{w}^{\mathcal{M}_{S}} T A$.

Now suppose $T A \notin w$. Case (i): $A \notin w$. Then by the induction hypothesis, $\forall_{w}^{\mathcal{M}_{S}} A$. Definition 1 gives us $\nvdash_{w}^{\mathcal{M}_{S}} T A$. Case (ii): $A \in w$. Consider the set $A \cup\{\neg B: T B \in w\}$. If that set is inconsistent, then there are $B_{1}, \ldots, B_{n}$ such that $T B_{1}, \ldots, T B_{n}$ are all in $w$ and $\vdash A \wedge \neg B_{1} \wedge \ldots \wedge \neg B_{n} \rightarrow \perp$. By RPL, $\vdash$ $A \rightarrow B_{1} \vee \ldots \vee B_{n}$. By RIM,$\vdash A \rightarrow\left(T\left(B_{1} \vee \ldots \vee B_{n}\right) \rightarrow T A\right)$. Since $A \in w$, $T\left(B_{1} \vee \ldots \vee B_{n}\right) \rightarrow T A \in w$. By $(\mathrm{T} 7), \vdash T B_{1} \wedge \ldots \wedge T B_{n} \rightarrow T\left(B_{1} \vee \ldots \vee B_{n}\right)$. Since $T B_{1}, \ldots, T B_{n}$ are all in $w, T\left(B_{1} \vee \ldots \vee B_{n}\right) \in w$. It follows that $T A \in w$, contrary to the assumption that $w$ is consistent. Hence the set $A \cup\{\neg B: T B \in w\}$ is consistent. By Lindenbaum's Lemma, it is a subset of some maximal $S$-consistent set $w^{\prime}$. By the induction hypothesis, $\vDash_{w^{\prime}}^{\mathcal{M}_{S}} A$. By the definition of $<_{S}$, $w^{\prime}<_{S} w$. Hence $\nvdash_{w}^{\mathcal{M}_{S}} T A$.

We are now in a position to prove the completeness of $\boldsymbol{C}$ with respect to the class of totality models.

Theorem 2. If $\vDash A$ then $\vdash A$.

Proof. Suppose $\forall A$. Then $\neg A$ is consistent in $C$. By Lindenbaum's Lemma, there is a maximally consistent set $w$ such that $\neg A \in w$. By the definition of $W_{C}$, $w \in W_{\boldsymbol{C}}$. With Lemma $3, \vDash_{w}^{\mathcal{M}_{C}} \neg A$. Hence $\not \models \neg A$.

Since a system is determined by a class of frames just in case it is sound and complete with respect to it, this establishes the following:

Theorem 3. System $\boldsymbol{C}$ is determined by the class of all totality frames.

\section{A system for partial order frames}

The system $\boldsymbol{C}$ is simple and elegant. As we have seen, it is sound and complete with respect to the class of all totality models. But we know from section 2 that in the intended model, $<$ is a strict partial order, i.e. asymmetric and transitive. So the question arises whether $\boldsymbol{C}$ is the strongest $L_{T}$-system that is sound with respect 
to the Chalmers-Jackson semantics. As it turns out, the answer is negative- $\boldsymbol{C}$ is not complete with respect to partial order frames. Unfortunately, the system that is sound and complete also appears to be considerably less simple and elegant than C.

It includes the following additional axiom schema:

(A3) $T A \rightarrow T(A \leftrightarrow(T B \rightarrow T(A \vee B)))$

(A3) does not wear its intuitive meaning on its sleeve. To understand it better, it is useful to temporarily adopt the primacy interpretation of $T$. With $H$ interpreted as "it has always been the case that", the following is equivalent, given the other logical principles, to (A3):

$\left(\mathrm{A} 3_{H}\right) T A \rightarrow H(T B \rightarrow T(A \vee B))$

$\left(\mathrm{A} 3_{H}\right)$ says that if $A$ is true for the first time, then it has always been the case that $B$ is true for the first time if and only if $A \vee B$ is true for the first time. On reflection, this is true - if $A$ is true for the first time, then it has always been false in the past, and hence $A \vee B$ cannot have become true before $B$.

Under the totality interpretation, but using the operator $O$ (Definition 2), (A3) is equivalent to the following:

$\left(\mathrm{A} 3^{\prime}\right) T A \rightarrow O(T B \rightarrow T(A \vee B))$

In words: if $A$ is total, then in every outstripped world in which $B$ is total, $A \vee B$ is also total.

If $\left(\mathrm{A} 3_{H}\right)$ appears obvious to us under the primacy interpretation, this is because we assume the relation of temporal precedence to be transitive. For we can show that (A3) corresponds - in the technical sense of that word (van Benthem (2001)) to the transitivity of $<$. Say that sentence $A$ defines a class of frames $S$ iff $A$ is valid on all frames in $S$ and invalid on all frames not in $S$. Then we can state the correspondence result as follows:

Theorem 4. Sentence (A3) defines the class of all transitive totality frames.

Proof. We first show that all instances of (A3) are valid in all transitive totality models. Suppose that $\mathcal{M}$ is transitive, and that $\models_{w}^{\mathcal{M}} T A$. Then $\models_{w}^{\mathcal{M}} A$. By (A2) and RPL,$\vdash T A \rightarrow(T B \rightarrow T(A \vee B))$. By the soundness result for $C, \vDash_{w}^{\mathcal{M}} T A \rightarrow$ $(T B \rightarrow T(A \vee B))$. Hence $\vDash_{w}^{\mathcal{M}} T B \rightarrow T(A \vee B)$. Hence $\vDash_{w}^{\mathcal{M}} A \leftrightarrow(T B \rightarrow T(A \vee B))$ 
It remains to show that whenever $w^{\prime}<w$, then $\vDash_{w^{\prime}}^{\mathcal{M}} \neg(A \leftrightarrow T B \rightarrow T(A \vee B))$. Since $\vDash_{w}^{\mathcal{M}} T A, \vDash_{w^{\prime}}^{\mathcal{M}} \neg A$. So the claim follows if we can show that $\vDash_{w^{\prime}}^{\mathcal{M}} T B \rightarrow$ $T(A \vee B)$. Suppose that $\vDash_{w^{\prime}}^{\mathcal{M}} \neg T(A \vee B)$. If $\vDash_{w^{\prime}}^{\mathcal{M}} \neg(A \vee B)$, then $\vDash_{w^{\prime}}^{\mathcal{M}} \neg T B$, and thus $\vDash_{w^{\prime}}^{\mathcal{M}} T B \rightarrow T(A \vee B)$. If $\vDash_{w^{\prime \prime}}^{\mathcal{M}} A \vee B$ for some $w^{\prime \prime}<w$, then by the transitivity of $<$, $w^{\prime \prime}<w$, and $\vDash_{w^{\prime \prime}}^{\mathcal{M}} \neg A$. Hence $\models_{w^{\prime \prime}}^{\mathcal{M}} B$, and thus $\models_{w^{\prime}}^{\mathcal{M}} \neg B$ and $\models_{w^{\prime}}^{\mathcal{M}} T B \rightarrow T(A \vee B)$.

Next, we show that (A3) fails on every frame that is not transitive. Suppose that $\mathcal{F}$ is not transitive. Then there are $w, w^{\prime}, w^{\prime \prime}$ such that $w^{\prime \prime}<w^{\prime}, w^{\prime}<w$, but not $w^{\prime \prime}<w$. Then $w^{\prime}$ is distinct from $w$ and from $w^{\prime \prime}$. Let $I(0)$ be $\left\{w, w^{\prime \prime}\right\}$, and $I(1)$ be $\left\{w^{\prime}\right\}$. Then $\vDash_{w}^{\mathcal{M}} T p_{0}$. Since $\vDash_{w^{\prime \prime}}^{\mathcal{M}} p_{0} \vee p_{1}, \forall_{w^{\prime}}^{\mathcal{M}} T\left(p_{0} \vee p_{1}\right)$. Since $\vDash_{w^{\prime}}^{\mathcal{M}} T p_{1}$, $\forall_{w^{\prime}}^{\mathcal{M}} T p_{1} \rightarrow T\left(p_{0} \vee p_{1}\right)$, and $\models_{w^{\prime}}^{\mathcal{M}} p_{0} \leftrightarrow T p_{1} \rightarrow T\left(p_{0} \vee p_{1}\right)$. Hence $\not_{w}^{\mathcal{M}} T\left(p_{0} \leftrightarrow T p_{1} \rightarrow\right.$ $\left.T\left(p_{0} \vee p_{1}\right)\right)$.

Define $\boldsymbol{C} 3$ to be the smallest system of total logic that contains all $L_{T}$-instances of (A3). By Theorem 4, some instances of (A3) fail on non-transitive totality frames. By the soundness result for $\boldsymbol{C}$, not all instances of (A3) are theses of the basic system $\boldsymbol{C}$, and hence $\boldsymbol{C} \mathbf{3}$ is distinct from $\boldsymbol{C}$.

As a corollary to Theorem 4, the soundness of $\boldsymbol{C} \mathbf{3}$ with respect to the class of transitive totality frame follows. I shall now turn to the task of establishing completeness. Lemma 3 applies to all systems of total logic, and hence to $\boldsymbol{C} \mathbf{3}$. As implicitly defined by clause 2 of Definition $3,<_{\boldsymbol{C} 3}$ is the relation that holds between maximally $C 3$-consistent sets of sentences $w^{\prime}$ and $w$ just in case $A \notin w^{\prime}$ whenever $T A \in w$. Establishing the transitivity of $<_{\boldsymbol{C} 3}$ would be enough to prove the completeness of $\boldsymbol{C} \mathbf{3}$ with respect to the class of transitive totality frames.

However, $<_{\boldsymbol{C} \mathbf{3}}$ is not transitive. It is a consequence of Definition 3.2 and Lemma 3 that if a world $w$ is inexhaustible in $\mathcal{M}_{S}$-i.e. such that $\not_{w}^{\mathcal{M}_{S}} T A$ for all $A \in L_{T}$ then $w$ outstrips every world in the canonical model: since $T A \in w$ is false for all $A$, the condition that $A \notin w^{\prime}$ whenever $T A \in w$ is vacuously satisfied by every $w^{\prime}$. It is a further consequence that every exhaustible world $v$ fails to outstrip itself: for some $A, \vDash_{v}^{\mathcal{M}_{S}} T A$ and hence both $T A \in v$ and $A \in v$. So if inexhaustible $w$ is outstripped by exhaustible $v$, we will have $v<_{S} w$ and $w<_{S} v$ but not $v<_{S} v$, in violation of transitivity.

To establish that $<_{\boldsymbol{C} 3}$ is not transitive, it remains to show that there is an inexhaustible $w$ and an exhaustible $v$ such that $w<_{\boldsymbol{C} 3} v$. In order to define such worlds - maximal $\boldsymbol{C}$ 3-consistent sets of sentences of $L_{T}$-I will use a (noncanonical) transitive totality model as an auxiliary device. Specifically, consider 
$\mathcal{N}=\langle W,<I\rangle$, with $W=\left\{w_{i}: i \in \mathbb{N}\right\},<=\left\langle w_{i}, w_{j}\right\rangle: i>_{\mathbb{N}} j$, and $I(0)=\left\{w_{0}\right\}$ and $I(j)=\emptyset$ for all $j \in \mathbb{N} \backslash\{0\}$. For $w \in W$, define $t(w):=\left\{A::_{w}^{\mathcal{N}} A\right\}$ (the "theory of $w$ "). Since $\mathcal{N}$ is a transitive totality model, and since $\boldsymbol{C} \mathbf{3}$ is sound with respect to such models, both $t\left(w_{0}\right)$ and $t\left(w_{1}\right)$ are maximally $\boldsymbol{C}$-consistent sets of $L_{T}$-sentences, and thus belong to the canonical model $\mathcal{M}_{\boldsymbol{C} \mathbf{3}}$. From Lemma 2 , and the fact that $w_{1}$ belongs to a homogeneous unending chain, it follows that $T A \notin t\left(w_{1}\right)$ for every $A \in L_{T}$. Hence $v<_{\boldsymbol{C} 3} t\left(w_{1}\right)$ for every $v \in W_{\boldsymbol{C} \mathbf{3}}$, and in particular, $t\left(w_{0}\right)<_{\boldsymbol{C} \mathbf{3}} t\left(w_{1}\right)$. Since $I(0)=\left\{w_{0}\right\}, \vDash_{w_{0}}^{\mathcal{N}} T p_{0}$, and hence $T p_{0} \in t\left(w_{0}\right)$. Consider any $A$ such that $T A \in t\left(w_{0}\right)$. Then $\models_{w_{0}}^{\mathcal{N}} T A$, and because $w_{1}<w_{0}, \nvdash_{w_{1}}^{\mathcal{N}} A$, and hence $A \notin t\left(w_{1}\right)$. Since $A$ was arbitrary, it follows that $t\left(w_{1}\right)<_{\boldsymbol{C} \mathbf{3}} t\left(w_{0}\right)$. But $T p_{0} \in t\left(w_{0}\right)$ and $p_{0} \in t\left(w_{0}\right)$ entail $t\left(w_{0}\right) \nless_{\boldsymbol{C} \mathbf{3}} t\left(w_{0}\right)$. So $<_{\boldsymbol{C} \mathbf{3}}$ is not transitive.

I shall now construct another canonical model where inexhaustible worlds only outstrip themselves. As before, they may be outstripped by exhaustible ones. ${ }^{10}$ For a set of sets of $L_{T}$-sentences $W$, let $W^{T}$ consist of those $w \in W_{S}$ such that $T A \in w$ for some $A$.

Definition 4. Given a system $S$ of total logic, let $\mathcal{M}_{S}^{\prime}$ be $\left\langle W_{S},<_{S}^{\prime}, I_{S}\right\rangle$, where

1. $W_{S}=\left\{w: w\right.$ a maximal $S$-consistent set of sentences of $\left.L_{T}\right\}$.

2. $<_{S}^{\prime}=\left\{\left\langle w^{\prime}, w\right\rangle\right.$ : either $w \in W_{S} \backslash W_{S}^{T}$ and $w=w^{\prime}$, or for all $A \in L_{T}, A \notin w^{\prime}$ whenever $T A \in w\}$.

3. $I_{S}(j)=\left\{w: p_{j} \in w\right\}$.

Again, it is obvious that $\mathcal{M}_{\mathcal{S}}^{\prime}$ is a totality model. The following result shows that it also deserves to be called a "canonical model".

Lemma 4. For every $w \in W_{S}$ and every $A, A \in w$ iff $\models_{w}^{\mathcal{M}^{\prime}}{ }_{S} A$.

Proof. This is proved just like Lemma 3, except that we need to cover the inductive step for $T A \notin w$ and $w \in W_{S} \backslash W_{S}^{T}$. If $A \notin w$, then $\not_{w}^{\mathcal{M}^{\prime}{ }_{S}} A$ by the induction hypothesis, and hence $\forall_{w}^{\mathcal{M}^{\prime}}{ }_{S} T A$. So suppose that $A \in w$. By the induction hypothesis, $\models_{w}^{\mathcal{M}^{\prime}}{ }_{S} A$. But since $w \in W_{S} \backslash W_{S}^{T}, w<w$, and hence $\forall_{w}^{\mathcal{M}^{\prime}}{ }_{S} T A$.

Theorem 5. If $\models^{\mathcal{F}}$ A for all transitive totality frames $\mathcal{F}$ then $\vdash_{C \mathbf{3}} A$.

\footnotetext{
${ }^{10}$ There is no reason to think that this new canonical model is in this respect like the intended model. Possible worlds that are not exhaustible by our language may well outstrip exhaustible ones.
} 
Proof. Suppose $\nvdash_{\boldsymbol{C} \mathbf{3}} A$. Then $\neg A$ is consistent in $\boldsymbol{C}$ 3. By Lindenbaum's Lemma, there is a maximally $\boldsymbol{C}$ 3-consistent set $w$ such that $\neg A \in w$. By the definition of $W_{\boldsymbol{C} \mathbf{3}}, w \in W_{\boldsymbol{C} \mathbf{3}}$. By Lemma $4, \not \nvdash_{w}^{\mathcal{M}^{\prime} \mathbf{C} 3} \neg A$.

It remains to show that $\mathcal{M}_{\boldsymbol{C} \mathbf{3}}^{\prime}$ is a transitive totality model. So suppose that $w^{\prime \prime}<_{\boldsymbol{C} \mathbf{3}}^{\prime} w^{\prime}$ and $w^{\prime}<_{\boldsymbol{C} \mathbf{3}}^{\prime} w$. Case (i): $w^{\prime} \in W_{S} \backslash W_{S}^{T}$. Then since $w^{\prime \prime}<_{\boldsymbol{C} \mathbf{3}}^{\prime} w^{\prime}$, $w^{\prime \prime}=w^{\prime}$, and thus $w^{\prime \prime}<_{\boldsymbol{C} \mathbf{3}}^{\prime} w$. Case (ii): $w^{\prime} \in W_{S}^{T}$. Then by the definition of $<_{\boldsymbol{C} \mathbf{3}}^{\prime}, w \in W_{S}^{T}$. Let $A$ be any sentence such that $T A \in w$. Then since $\vdash_{\boldsymbol{C} \mathbf{3}}$ (A3), $T\left(A \leftrightarrow(T B \rightarrow T(A \vee B)) \in w\right.$, for every $B$. Since $w^{\prime}<_{C \mathbf{3}}^{\prime} w, \neg(A \leftrightarrow$ $(T B \rightarrow T(A \vee B))) \in w^{\prime}$ and $\neg A \in w^{\prime}$. Given that $w^{\prime}$ is closed under RPL, $T B \rightarrow T(A \vee B) \in w^{\prime}$, for every $B$. By hypothesis $w^{\prime} \in W_{S}^{T}$, such that there is a sentence $C$ such that $T C \in w^{\prime}$. Hence $T(A \vee C) \in w^{\prime}$. Since $w^{\prime \prime}<w^{\prime}$ and $w^{\prime} \in W_{S}^{T}, \neg(A \vee B) \in w^{\prime \prime}$. Since $w^{\prime \prime}$ is closed under RPL, $\neg A \in w^{\prime \prime}$. Since this holds for every $A$ such that $T A \in w$, and since $w \in W_{S}^{T}, w^{\prime \prime}<w$.

Hence, from Theorems 4 and 5:

Theorem 6. System $\boldsymbol{C} \mathbf{3}$ is determined by the class of transitive totality frames.

The frame of the intended model is not only transitive, but also asymmetricit is a partial order frame. This prompts the question whether the asymmetry constraint allows us to deploy an even stronger system of total logic. The answer is negative: if a sentence of $L_{T}$ is invalid in some transitive totality frame, it is also invalid in some partial order frame.

I shall prove the completeness of $\boldsymbol{C} \mathbf{3}$ for partial order frames by applying a version of the technique known as "bulldozing". For a simple example of how this works, consider a model with just two worlds, $w$ and $v$, where $w<v$ and $v<w$. The bulldozing technique turns these into infinitely "copies" $w_{0}, w_{1}, \ldots$ of $w$ and $v_{0}, v_{1}, \ldots$ of $v$, where $w_{0}<w_{1}$ and $w_{0}<v_{1}$ as well as $v_{0}<v_{1}$ and $v_{0}<w_{1}$ (and likewise $w_{1}<w_{2}, w_{1}<v_{2}, v_{1}<v_{2}, v_{1}<w_{2}$, etc). This way we turn symmetric models into asymmetric ones. ${ }^{11}$

Formally, with $w_{i}$ abbreviating $\langle w, i\rangle$ :

Definition 5. For a totality model $\mathcal{M}=\langle W,<, P\rangle$, define $\overline{\mathcal{M}}=\langle\bar{W}, \overline{<}, \bar{I}\rangle$ as follows:

1. $\bar{W}=W \times \mathbb{N}$

\footnotetext{
${ }^{11}$ For a general account of bulldozing, see Blackburn et al. (2001, p. 220-222), for example.
} 
2. $v_{i} \overline{<} w_{j}=_{d f} v<w$ and $i>_{\mathbb{N}} j$

3. $\bar{I}(j)=I(j) \times \mathbb{N}$

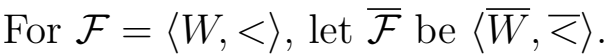

Lemma 5. If $\mathcal{F}$ is a transitive totality frame, $\overline{\mathcal{F}}$ is a partial order frame.

Proof. For transitivity, suppose that $w_{i} \overline{<} w_{j}^{\prime}$ and $w_{j}^{\prime} \overline{<} w_{k}^{\prime \prime}$. Then $w<w^{\prime}$ and $w^{\prime}<$ $w^{\prime \prime}$, and since $<$ is transitive, $w<w^{\prime \prime}$. Also $i>j$ and $j>k$, and by the transitivity of $>_{\mathbb{N}}, i>k$. Hence $w_{i}<w_{k}^{\prime \prime}$.

For antisymmetry, suppose that $w_{i} \overline{<} w_{j}^{\prime}$. Then $i>_{\mathbb{N}} j$. By the asymmetry of $>_{\mathbb{N}}, j \ngtr_{\mathbb{N}} i$. Hence it is not the case that $w_{j}^{\prime} \overline{<} w_{i}$.

Lemma 6. For all $A \in L_{T}, j \in \mathbb{N}$ and every totality model $\mathcal{M}=\langle W,<, I\rangle, \vDash_{w}^{\mathcal{M}} A$ iff $\vDash{ }_{w_{j}}^{\overline{\mathcal{M}}} A$

Proof. By induction on the complexity of $A$.

For atomic $p_{j}, \models_{w}^{\mathcal{M}} p_{j}$ iff $w \in I(j)$ iff $w_{j} \in I(i) \times \mathbb{N}$ iff $w_{j} \in I(j)$ iff $\vDash_{w_{j}}^{\overline{\mathcal{M}}} p_{j}$. The induction steps for the usual propositional connectives are routine.

Now suppose that $\models_{w}^{\mathcal{M}} T A$. Then $\vDash_{w}^{\mathcal{M}} A$. By the induction hypothesis, $\vDash_{w_{i}}^{\overline{\mathcal{M}}} A$. Let $w_{k}^{\prime} \overline{<} w_{i}$. Then $w^{\prime}<w$. Since $\models_{w}^{\mathcal{M}} T A, \nvdash_{w^{\prime}}^{\mathcal{M}} A$. By the induction hypothesis, $\forall_{w_{k}^{\prime}}^{\overline{\mathcal{M}^{\prime}}} A$. Hence $\models_{w_{j}}^{\overline{\mathcal{M}}} T A$.

For the other direction, suppose $\nvdash_{w}^{\mathcal{M}} T A$. Case (i): $\nvdash_{w}^{\mathcal{M}} A$. Then by the induction hypothesis, $\forall_{w_{i}}^{\overline{\mathcal{M}}} A$, and hence $\nvdash_{w_{i}}^{\overline{\mathcal{M}}} T A$. Case (ii): $\models_{w^{\prime}}^{\mathcal{M}} A$ for some $w^{\prime}$ with $w^{\prime}<w$. B y the induction hypothesis, $\models_{w_{j}^{\prime}}^{\overline{\mathcal{M}}} A$ for every $j \in \mathbb{N}$. Pick $k$ such that $k>_{\mathbb{N}} i$. Then $w_{k}^{\prime} \overline{<} w_{i}$. Hence $\not_{w_{i}}^{\overline{\mathcal{M}}} T A$.

Theorem 7. If $\vDash^{\mathcal{F}}$ A for all partial order frames $\mathcal{F}$ then $\vdash_{C \mathbf{3}} A$.

Proof. Suppose $\forall_{\boldsymbol{C} 3} A$. Then by Theorem $5, \forall_{w}^{\mathcal{M}} A$ for some transitive totality model $\mathcal{M}=\langle W,<, I\rangle$ and some $w \in W$. By Lemma $6, \not \nvdash_{w_{j}}^{\overline{\mathcal{M}}} A$. By Lemma $5, \overline{\mathcal{M}}$ is a partial order model. Hence $\not^{\overline{\mathcal{F}}} A$ for the partial order frame $\overline{\mathcal{F}}=\langle\bar{W}, \overline{<}, \bar{I}\rangle$.

So we have a second determination result for $\boldsymbol{C} \mathbf{3}$ :

Theorem 8. System $\boldsymbol{C} \mathbf{3}$ is determined by the class of partial order frames. 


\section{$6 \quad$ Further principles and frame conditions}

On the Chalmers-Jackson account, outstripping is a strict partial order - as we have seen, only relatively uncontroversial assumptions about parthood and duplication are needed to establish this. In the last section, the system $\boldsymbol{C} \mathbf{3}$ was shown to be sound and complete for strict partial order frames. This falls short of establishing $\boldsymbol{C} 3$ as the correct total logic, though, even given the Chalmers-Jackson account of outstripping. For all that has been said, that account may implicitly impose further conditions on outstripping, which validate principles that are not theses of C3.

In the temporal case, which is many respects analogous, the assumption of linearity is quite plausible: any two instants stand in the relation of precedence, in some order. But the metaphysical assumption that outstripping is linear has no plausibility whatsoever. A world consisting of a hydrogen atom outstrips both a world consisting of a lone proton and a world consisting of a lone electron, but neither of the latter two outstrips the other.

Some other additional frame conditions may be less implausible, though. Instead of starting with the frame conditions, I shall formulate prima facie plausible principles, and then ask what frame conditions they correspond to.

The first principle to be considered, (A4), says that $\neg \perp$ (sometimes written $\top$ ) is not total.

(A4) $\neg T \neg \perp$

The idea is clear: a tautology cannot possibly be a comprehensive truth.

Another prima facie plausible principle concerns iteration. We have seen that the principle $T A \rightarrow T T A$ (T1), reminiscent of $\mathrm{S} 4$, is a thesis already of the base logic $C$. The principle $\neg T A \rightarrow T \neg T A$, reminiscent of S5, is highly implausible$\neg T A$ does not tell us much, not even whether $A$ is true or not. But for that very reason, $\neg T A \rightarrow \neg T \neg T A$ appears promising: $\neg T A$ contains so little information that it can hardly be total. Since $\vdash_{C} T A \rightarrow \neg T \neg T A$ (from (A1) and contraposition), adding the schema $\neg T A \rightarrow \neg T \neg T A$ to a system of total logic has the same effect as adding the simpler (A5):

(A5) $\neg T \neg T A$

This principle says that no negative totality statement can be total. 
As it turns out, (A4) is a thesis of the system $\boldsymbol{C 5}$ (defined as the smallest system of total logic that includes all $L_{T}$-instances of (A5)). For by (A1), $\vdash_{\boldsymbol{C 5}} T A \rightarrow$ $\neg T \neg A$. By $(\mathrm{A} 0), \vdash_{\boldsymbol{C 5}} \neg T A \rightarrow \neg \perp$, and by RIM, $\vdash_{C \mathbf{5}} \neg T A \rightarrow(T \neg \perp \rightarrow T \neg T A)$. By RPL, $\vdash_{C 5} \neg T A \rightarrow(\neg T \neg \neg T A \rightarrow \neg T \neg \perp)$. The result follows from (A5) and RPL.

If we identify a system, as is customary, with the set of its theses, then $\boldsymbol{C 4} \subseteq$ $C 5$, and $C 45=C 5$. It also follows that $C 34 \subseteq C 35=C 345$. (For easy reference, the results about inclusion among systems proved in this section are diagrammatically represented in Figure 1, below. An extension of a system is reached upwards along the lines.)

Every instance of (A5) is a thesis of $\boldsymbol{C 3 4}$. Using (T1), (A2), and RRep, $\vdash_{\boldsymbol{C}}$ $T A \wedge T(\neg T A \vee A) \rightarrow T \neg \perp$. By $(\mathrm{A} 4), \vdash_{C 4} \neg(T A \wedge T(\neg T A \vee A))$. With RPL, it follows that $\vdash_{\boldsymbol{C 4}}(T A \rightarrow T(\neg T A \vee A)) \leftrightarrow \neg T A$. By an instance of (A3), $\vdash_{\mathbf{C 3 4}} T \neg T A \rightarrow T\left(\neg T A \leftrightarrow\left(T A \rightarrow T(\neg T A \vee A) . \quad\right.\right.$ By RRep, $\vdash_{\mathbf{C 3 4}} T \neg T A \rightarrow$ $T(\neg T A \leftrightarrow \neg T A)$. Since $\vdash_{C}(\neg T A \leftrightarrow \neg T A) \leftrightarrow \neg \perp, \vdash_{C 34} T \neg T A \rightarrow T(\neg \perp)$. Applying (A4) and RPL, $\vdash_{C \mathbf{3 4}} \neg T \neg T A$.

Together with the earlier result, this entails $\boldsymbol{C 3 4}=\boldsymbol{C 3 5}=\boldsymbol{C 3 4 5}$.

To establish the distinctness of certain systems, we will use model-theoretical tools. Say that a frame $\mathcal{F}=\langle W,<\rangle$ is serial if for all $w \in W$, there is $w^{\prime}$ such that $w^{\prime}<w$. In a serial frame, no world is minimal.

Theorem 9. Sentence (A4) is defined by the class of serial frames.

Proof. To show that (A4) is valid in every serial frame $\mathcal{F}$, pick any $w \in W$. By seriality, there is $w^{\prime}$ with $w^{\prime}<w$. Then $\vDash_{w}^{\mathcal{F}} \neg \perp$, and hence $\models_{w}^{\mathcal{F}} \neg T \neg \perp$. Now suppose that $\mathcal{F}$ is not serial. Then there is $w \in W$ such that $w^{\prime} \nless w$ for all $w^{\prime} \in W$. Hence $\vDash_{w}^{\mathcal{F}} T \neg \perp$.

Let $\mathcal{F}$ be an irreflexive frame consisting of one world. Since $\mathcal{F}$ is transitive, every thesis of $\boldsymbol{C} \mathbf{3}$ is valid in it. But since it is not serial, (A4) is not. Hence $\boldsymbol{C 4} \nsubseteq \boldsymbol{C}$ 3. Since some serial frames - on which every thesis of $\boldsymbol{C 4}$ is valid - are not transitive, the converse result, $\boldsymbol{C} \mathbf{3} \nsubseteq \boldsymbol{C 4}$, also follows.

Determination results are now straightforward.

Theorem 10. System $\boldsymbol{C 4}$ is determined by the class of all serial frames.

Proof. It is enough to show that the canonical model $\mathcal{M}_{\boldsymbol{C 4}}$ (Definition 3) is serial. 
For every $w \in W_{\boldsymbol{C 4}}, \neg T(\neg \perp) \in w$. By Lemma $3, \models_{w}^{\mathcal{M}_{\boldsymbol{C} 4}} \neg T(\neg \perp)$. Since $\vDash_{w}^{\mathcal{M}_{\boldsymbol{C} 4}} \neg \perp$, there is $w^{\prime} \in W_{\boldsymbol{C} 4}$ such that $w^{\prime}<w$.

Theorem 11. System $C 34(=C 35)$ is determined by the class of all serial partial order frames.

Proof. We first show that the canonical model $\mathcal{M}_{\boldsymbol{C} \mathbf{3 4}}^{\prime}$ (Definition 4), already shown to be transitive, is serial. For every $w$ in the model, $\neg T(\neg \perp) \in w$. By Lemma 4 , $\vDash^{\mathcal{M}_{C 34}^{*}} \neg T(\neg \perp)$. Since $\vDash^{\mathcal{M}_{C 34}^{*}} \neg \perp$, there is a world $w^{\prime}$ such that $w^{\prime}<w$.

Next, we assume that $A$ fails in a transitive and serial model $\mathcal{M}=\langle W,<, I\rangle$. In the light of Lemma 6 , it is enough to verify that the operation of bulldozing of Definition 5 preserves seriality. Pick $w \in \bar{W}$. Then $w=\langle v, n\rangle$, with $v \in W$ and $n \in \mathbb{N}$. By the seriality of $<$, there exists $v^{\prime} \in W$ such that $v^{\prime}<v$. Define $w^{\prime}$ as $\left\langle v^{\prime}, n+1\right\rangle$. Then $w^{\prime} \overline{<} w$. So $\overline{<}$ is serial.

While (A4) corresponds to seriality, (A5) corresponds to a a closely related condition. Let a world $w$ be doubly outstripping in $\mathcal{F}$ if there are $w^{\prime}, w^{\prime \prime}$ such that $w^{\prime \prime}<w^{\prime}, w^{\prime}<w$, and $w^{\prime \prime}<w$. Further, say that $A$ is valid in a world $w$ in frame $\mathcal{F}$ if $\models_{w}^{\mathcal{M}} A$ for every model $\mathcal{M}$ on frame $\mathcal{F}$.

Lemma 7. Sentence (A5) is valid in world $w$ in frame $\mathcal{F}$ iff $w$ is doubly outstripping in $\mathcal{F}$.

Proof. First, I show that (A5) is valid in every world $w$ that is doubly outstripping in $\mathcal{F}$. There are $w^{\prime}, w^{\prime \prime}$ with $w^{\prime}<w, w^{\prime \prime}<w$, and $w^{\prime \prime}<w^{\prime}$. If $\not_{w^{\prime}}^{\mathcal{M}} A$, then $\models_{w^{\prime}}^{\mathcal{M}} \neg T A$, and thus $\vDash_{w}^{\mathcal{M}} \neg T(\neg T A)$. If $\not_{w^{\prime \prime}}^{\mathcal{M}} A$, we reason in the same way. So assume $\models_{w^{\prime}}^{\mathcal{M}} A$ and $\not \nvdash_{w^{\prime \prime}}^{\mathcal{M}} A$. Since $w^{\prime \prime}<w^{\prime}, \models_{w^{\prime}}^{\mathcal{M}} \neg T A$, and since $w^{\prime}<w, \vDash_{w}^{\mathcal{M}} \neg T(\neg T A)$.

Now suppose that $w$ is not doubly outstripping in $\mathcal{F}$. Then there is $w \in W$ such that for all $w^{\prime}, w^{\prime \prime} \in W$, if $w^{\prime}<w$ and $w^{\prime \prime}<w$, then neither $w^{\prime \prime}<w^{\prime}$ nor $w^{\prime}<w^{\prime \prime}$. Note that in particular, $w \nless w$. Define $I(0)=\{v: v<w\}$. Then $\vDash_{w}^{\mathcal{M}} \neg p_{0}$, and thus $\vDash_{w}^{\mathcal{M}} \neg T p_{0}$. Consider any $w^{\prime}$ with $w^{\prime}<w$. By the definition of $I$, $\vDash_{w^{\prime}}^{\mathcal{M}} p_{0}$. Now consider any $w^{\prime \prime}$ such that $w^{\prime \prime}<w^{\prime}$. Then $w^{\prime \prime} \nless w$, and thus $\nvdash_{w^{\prime \prime}}^{\mathcal{M}} p_{0}$. Hence $\models_{w^{\prime}}^{\mathcal{M}} T p_{0}$, and thus $\not{ }_{w}^{\mathcal{M}} T \neg T p_{0}$.

Say that a frame $\mathcal{F}$ is bi-serial if every $w \in W$ is doubly outstripping. The following is a straightforward consequence of Lemma 7 :

Theorem 12. Sentence (A5) is defined by the class of bi-serial frames. 
Clearly, every bi-serial frame is serial. The converse also holds in the class of transitive frames. For suppose that $<$ is transitive and serial, and let $w \in W$. By seriality, there is $w^{\prime} \in W$ such that $w^{\prime}<w$. By seriality again, there is $w^{\prime \prime} \in W$ such that $w^{\prime \prime}<w^{\prime}$. By transitivity, $w^{\prime \prime}<w$. So $<$ is serial.

Since not every serial frame is bi-serial, Theorem 12 and Theorem 9 together entail that (A5) is not a thesis of $\boldsymbol{C 4}$, and hence that $\boldsymbol{C 5} \neq \boldsymbol{C 4}$. It is likewise easy to verify that $\boldsymbol{C} \mathbf{3} \nsubseteq \boldsymbol{C 5}$. Clearly, $C \mathbf{3} \subseteq C \mathbf{3 4}$, so $\boldsymbol{C 3 4}$ is distinct from $\boldsymbol{C 5}$.

The intended frame, which is transitive, will be bi-serial provided that it is serial. But is it serial? It would appear that it is not, i.e. that there are possible worlds that do not outstrip any other possible world. One relevant example is a point-sized possible world: since it has no proper spatial parts, no possible world duplicates any of its proper spatial parts.

A defender of seriality has three responses. First, she may hold that spacetime has, necessarily, only regions as parts, and no points. ${ }^{12}$ Second, she may hold that while extended worlds have point-sized parts, no possible world is itself pointsized. A third defence of seriality turns on the observation that the ChalmersJackson account is only adequate if things have non-spatiotemporal parts. These non-spatiotemporal parts may be properties - as I shall assume here - or bearers of properties. So, for example, a point particle may have a certain determinate of mass as a proper part. According to a theory of quantities due to David M. Armstrong, mass determinates have smaller mass determinates as proper parts. ${ }^{13}$ But presumably, there is no zero-mass part. Given that there is no smallest nonzero mass, every mass part of a point particle would seem to have a proper part that duplicates another one-particle world. If the case of mass is representative for all property parts, then every typical possible world outstrips some other possible world. This is, admittedly, a big "if" - but my present aim is only to discuss the consequences of certain metaphysical views for the logic of totality, not to evaluate those metaphysical views. ${ }^{14}$

But even if every typical possible world outstrips some other worlds, the intended frame may still fail to be serial - namely, if there are also untypical worlds that do not outstrip any world. Prime candidates here are so-called "empty

\footnotetext{
${ }^{12}$ For discussion, see Arntzenius (2008).

${ }^{13}$ This is somewhat simplified, but not in a way that would affect the present discussion.

${ }^{14}$ For reasons against generalizing from the case of mass, see the critical discussion of Armstrong's account in Eddon (2007).
} 
worlds". There has been a large debate about whether there are such things. ${ }^{15}$ Offhand, it seems that there ought to be, since it is not necessary that there is something rather than nothing. However, some theorists of possible worldsnotably David Lewis - had trouble making room for such things in their theories. If possible worlds are concrete things, how can there be an empty one?

An empty world leads to further theoretical difficulties in the context of the Chalmers-Jackson account of outstripping. To recall, the account reads:

CJ-Outstripping $w$ outstrips $w^{\prime}$ iff some proper part of $w$ duplicates $w^{\prime}$, but no proper part of $w^{\prime}$ duplicates $w$.

This clearly has the result that if there is an empty world-which has no proper parts - then that world does not outstrip any other one, in violation of seriality. So far, so good. But it also seems to lead to the result that an empty world is not outstripped by any world, not even non-empty ones. What part of a non-empty world is duplicated by an empty one? At best a so-called "null individual". But standard mereology does not countenance null individuals. So it seems that on the Chalmers-Jackson account, empty worlds are neither in the domain nor the co-domain of the relation of outstripping. ${ }^{16}$

The problem may require a small modification of the Chalmers-Jackson account of outstripping.

CJ-Outstripping* $w$ outstrips $w^{\prime}$ iff $w$ is non-empty and $w^{\prime}$ empty, or some proper part of $w$ duplicates $w^{\prime}$, but no proper part of $w^{\prime}$ duplicates $w$.

This disjunctive account admittedly looks somewhat ad hoc. It would take us too deep into mereology to discuss alternative solutions.

A metaphysican who accepts empty possible worlds and CJ-Outstripping* has to reject (A4) and (A5) as invalid. But suppose that she thinks that seriality holds among the non-empty worlds - that every non-empty outstrips another non-empty world. She can then still accept a system that properly extends $\boldsymbol{C}$, as I will now show.

Given CJ-Outstripping*, and supposing that there is one and only one empty world, mereologically atomic worlds will outstrip exactly one world. Let a frame

\footnotetext{
${ }^{15}$ Coggins (2010) is a book-length treatment, with references.

${ }^{16}$ Thanks to Alex Skiles for raising this issue.
} 
$\langle W,<\rangle$ be non-atomic if every $w \in W$ is either doubly outstripping or else is minimal (i.e. such that $w^{\prime} \nless w$ for every $w^{\prime} \in W$ ). Consider now an axiom schema that is equivalent to the material conditional with (A4) as the antecedent and (A5) as the consequent:

(A6) $T \neg T A \rightarrow T \neg \perp$

Clearly, $\boldsymbol{C 6} \subseteq \boldsymbol{C 5}$ - every thesis of the system $\boldsymbol{C 6}$ is also a thesis of $\boldsymbol{C 5}$.

Theorem 13. Sentence (A6) defines the class of non-atomic frames.

Proof. If $w$ is minimal in $\mathcal{F}$, then for every model $\mathcal{M}$ on $\mathcal{F}, \vDash_{w}^{\mathcal{M}} T \neg \perp$, and hence $\vDash_{w}^{\mathcal{M}} T \neg T A \rightarrow T \neg \perp$. If $w$ is doubly outstripping, then by Lemma 7 , in $\mathcal{F}$, then for every model $\mathcal{M}$ on $\mathcal{F}$, and every $A \in L_{T}, \vDash_{w}^{\mathcal{M}} \neg T \neg T A$, and hence $\vDash_{w}^{\mathcal{M}} T \neg T A \rightarrow T \neg \perp$. So (A6) is valid in that class. Suppose now that $\mathcal{F}=\langle W,<\rangle$ is not a non-atomic frame. Then there is $w \in W$ that is neither doubly outstripping nor minimal. By 7 , there is a model $\mathcal{M}$ on $\mathcal{F}$ such that $\vDash_{w}^{\mathcal{M}} T \neg T p_{0}$. But since $w$ is not minimal in $\mathcal{F}, \vDash_{w}^{\mathcal{M}} \neg T \neg \perp$. Hence an instance of (A6) fails at $w$ in $\mathcal{M}$.

Since (A6) is not valid on transitive frames, it is not a thesis of $\boldsymbol{C} \mathbf{3}$. It follows that $\boldsymbol{C 6} \nsubseteq \boldsymbol{C 3}$ and $\boldsymbol{C 3 6} \nsubseteq \boldsymbol{C 3}$. Conversely, we have $\boldsymbol{C 3 6} \nsubseteq \boldsymbol{C 6}$, due to the existence of non-atomic frames that fail to be transitive. Moreover, some nonatomic frames are not serial, and vice versa, such that $\boldsymbol{C 6} \nsubseteq \mathbb{C 4}$ and $\boldsymbol{C 4} \nsubseteq \boldsymbol{C 6}$.

With $\boldsymbol{C 6}$ and $\boldsymbol{C 3 6}$, all the systems to be discussed in this paper have been introduced. Their relationships are summarized in following Figure 1. To recall, the candidates for the right system of total logic seem to be $\boldsymbol{C} \boldsymbol{3}$ and its extensions $C 36$ and $C 34$.

I shall conclude the paper by proving the expected determination results for C36:

Theorem 14. System $C 36$ is determined by the class of transitive and non-atomic frames.

Proof. Soundness has in effect already been established. In light of the proof of Theorem 5, verifying that $\mathcal{M}^{\prime}$ (Definition 4) is non-atomic is sufficient for establishing the completeness part. Suppose that $w \in W_{\boldsymbol{C} \mathbf{3 6}}$ is not minimal. Then $\vDash_{w}^{\mathcal{M}^{\prime}} \neg T \neg \perp$. By Lemma $4, \neg T \neg \perp \in w$. Since $T \neg T \neg \perp \rightarrow T \neg \perp$ is an instance of (A6), it is a member of $w$. Hence $\neg T \neg T \neg \perp \in w$. By Lemma $4, \models_{w}^{\mathcal{M}^{\prime}} \neg T \neg T \neg \perp$, and 
Figure 1: Systems extending $\boldsymbol{C}$

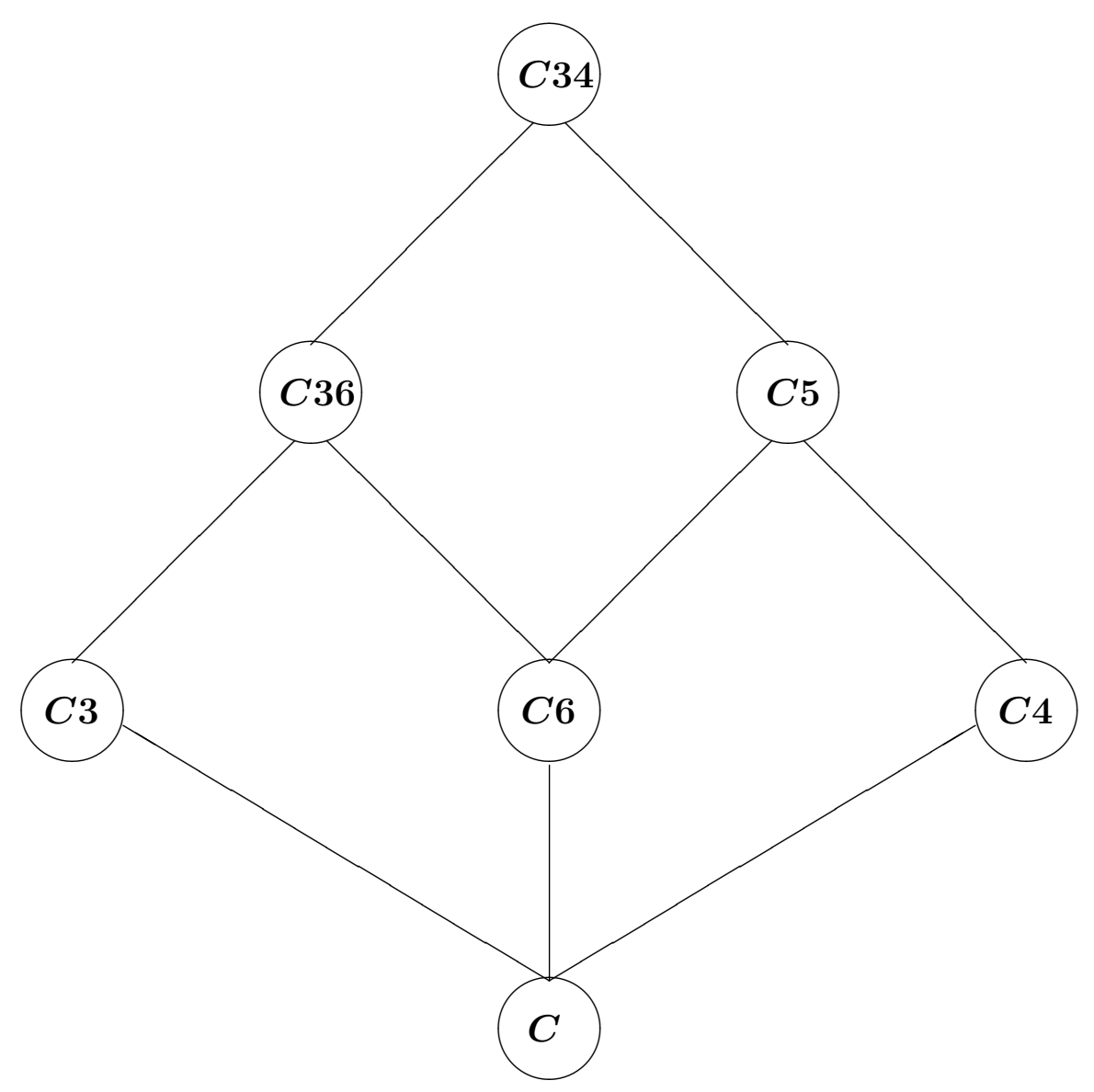


there is thus $w^{\prime} \in W_{\mathbf{C 3 6}}$ with $w^{\prime}<^{\prime} w$ and $\models_{w^{\prime}}^{\mathcal{M}^{\prime}} \neg T \neg \perp$. Then $w^{\prime}$ is not minimal, and we can repeat the argument to show that there is $w^{\prime \prime} \in W_{\boldsymbol{C} 36}$ with $w^{\prime \prime}<^{\prime} w^{\prime}$. Since $<^{\prime}$ is transitive, $w^{\prime \prime}<w$. It follows that $w$ is doubly outstripping and $\mathcal{M}^{\prime}$, and hence that the frame of $\mathcal{M}^{\prime}$ is non-atomic as well as transitive.

Since bulldozing (Definition 5) maps minimal worlds to minimal worlds and doubly outstripping worlds to doubly outstripping worlds, the following also holds:

Theorem 15. System $\mathbf{C} 36$ is determined by the class of non-atomic partial order frames.

\section{References}

David M. Armstrong. A Combinatorial Theory of Possibility. Cambridge University Press, 1989.

Frank Arntzenius. Gunk, topology and measure. In Dean W. Zimmerman, editor, Oxford Studies in Metaphysics, pages 225-247. Oxford University Press, Oxford, 2008.

Patrick Blackburn, Maarten de Rijke, and Yde Venema. Modal Logic. Cambridge Tracts in Computer Science. Cambridge University Press, Cambridge, 2001.

David J. Chalmers. The two-dimensional argument against materialism. In The Character of Consciousness, pages 141-192. Oxford University Press, Oxford, 2010.

David J. Chalmers. Constructing the World. Oxford University Press, Oxford, 2012.

David J. Chalmers and Frank Jackson. Conceptual analysis and reductive explanation. The Philosophical Review, 110:315-361, 2001.

Geraldine Coggins. Could There Have Been Nothing? Against Metaphysical Nihilism. Palgrave Macmillan, 2010.

Maya Eddon. Armstrong on quantities and resemblance. Philosophical Studies, 136:385-404, 2007. 
Frank Jackson. Armchair metaphysics. In John O'Leary-Hawthorne and Michaelis Michael, editors, Philosophy in Mind. Kluwer, Dordrecht, 1994.

Frank Jackson. From Metaphysics to Ethics. OUP, 1998.

John Lemmon and Dana Scott. An introduction to modal logic. Blackwell, Oxford, 1977.

Johan van Benthem. Correspondence theory. In D.M. Gabbay and F. Guenthner, editors, Handbook of Philosophical Logic, volume 3, pages 325-408. Kluwer Academic Publishers, Dordrecht, 2nd edition, 2001.

Achille Varzi. Mereology. In Edward N. Zalta, editor, Stanford Encyclopedia of Philosophy. Winter 2012 edition, 2012. 